\title{
Voltage-dependence of magnetoresistance in ferromagnetic tunneling junctions: a rigorous free electron model study
}

\author{
S.S. Liu, G.Y. Guo* \\ Department of Physics, National Taiwan University, 1 Sec. 4, Roosevelt Road, Taipei, Taiwan 106, ROC
}

\begin{abstract}
Spin-dependent tunneling current and tunneling magnetoresistance (MR) of ferromagnetic tunneling junctions have been calculated within the free electron model by solving rigorously the 3D Schrödinger equation for different bias voltages and barrier widths. It is found that in general, the MR would decrease with voltage and become negative at some large voltages, a trend in agreement with both earlier and recent experiments. Also, it is revealed that for certain barrier widths, the MR would oscillate with bias voltage, a prediction to be confirmed by experiments. (C) 2000 Elsevier Science B.V. All rights reserved.
\end{abstract}

Keywords: Magnetoresistance; Magnetic tunneling junction

Large magnetoresistance (MR) observed in ferromagnet-insulator-ferromagnet $(\mathrm{F} / \mathrm{I} / \mathrm{F})$ tunneling junctions has attracted much attention in recent years mainly because of its fundamental importance in transport physics and its potential applications in magnetic sensors and memory devices. The MR ratio is defined by

$\mathrm{MR}=\frac{\Delta G}{G}=\frac{G_{\mathrm{p}}-G_{\mathrm{ap}}}{G_{\mathrm{p}}}$,

where $G_{\mathrm{p}}$ is the conductance of the tunneling junction when the two ferromagnets are parallel, and $G_{\text {ap }}$, when the two ferromagnets are antiparallel. The MR first observed by Julliere [1] was $14 \%$ in the $\mathrm{Co} / \mathrm{Ge} / \mathrm{Co}$ junction at $4.2 \mathrm{~K}$ under nearly zero bias. Julliere also proposed a simple model which relates the positive MR only to the product of the spin-polarizations of the two ferromagnets. [1] The simple model of Julliere was further developed by Slonczewski [2] who used a quasi-one-dimensional free electron model with the assumption of large barrier widths and zero bias. In recent experiments, a strong decrease in the MR as the applied voltage

\footnotetext{
*Corresponding author. Tel.: + 886-2-23626937; fax: + 8862-23639984.

E-mail address: gyguo@phys.ntu.edu.tw (G.Y. Guo)
}

increases was observed $[3,4]$. This would limit the application of the MR tunneling junctions. Interestingly, Sharma et al. [5] very recently found a negative MR ratio at certain applied voltages. This observed negative MR ratio appears to be in contradiction to Julliere's simple model on the MR effect.

Therefore, it seems imperative to investigate theoretically the MR under a finite bias. Indeed, three groups have recently discussed the finite bias effects [6-8]. However, approximations similar to Ref. [2] were also made by Chui [6] and Bratkovsky [7]. Zhang et al. [8], on the other hand, solved rigorously the quasi-one-dimensional free electron model by using a $2 \times 2$ transfer matrix method. Nevertheless, Zhang et al. [8] used some unphysical parameters especially too small barrier height that would make the junctions short-circuited.

In this work, we consider a $\mathrm{F} / \mathrm{I} / \mathrm{F}$ junction grown in the $z$-direction and model the junction by solving the full three-dimensional (3D) single-electron Schrödinger equation which for the longitudinal wave functions in the $j$ th region is

$\left[-\frac{\hbar^{2}}{2 m_{j}^{*}} \frac{d^{2}}{d z^{2}}+\frac{h^{2} k_{t}^{2}}{2 m_{j}^{*}}+V_{j}-h_{j} \sigma_{j}-\mathrm{e} F_{j} z\right] \psi(z)=E \psi(z)$,

where the constants $V_{j}, h_{j}$ and $F_{j}$ are the band edge, exchange field and external electric field in the $j$ th region, 
respectively. $k_{\mathrm{t}}$ is the transverse wave vector, and the Pauli spin matrix $\sigma$ is taken along the local magnetization direction $\mathbf{h}_{j}$ in the $j$ th region. By matching both $\psi(z)$ and $\left[\psi^{\prime}(z) / m^{*}\right]$ at the layer interfaces using a $4 \times 4$ transfer matrix method, we are able to solve the single electron Schrödinger equation for the whole junction and calculate the physical quantities such as transmission coefficient $T\left(E_{z}, E_{\mathrm{t}}\right)$. The tunneling current density $J$ is then obtained from the transmission coefficient by integration over both the longitudinal energy $\left(E_{z}\right)$ and the transverse energy $\left(E_{\mathrm{t}}\right)$ as

$J=\frac{e}{2 \pi^{2} h} \int \mathrm{d} E_{z}[f(E)-f(E+e V)] \iint \mathrm{d}^{2} k_{\mathrm{t}} T\left(E_{z}, E_{\mathrm{t}}\right)$,

where the Fermi function $f(E)$ at zero temperature is assumed here.

Unlike Slonczewski [2] and Zhang et al. [8] who considered only a quasi-one-dimensional Hamiltonian, we also take into account the transverse (in-plane) motion of the electrons as well as the region-dependent effective mass. Note that the effective mass in the barrier is often different from the free electron mass. When taking both the transverse motion and the space-dependent effective mass of the electron into account, the transmission coefficient can have a pronounced dispersion in the transverse wave vector space [9], in contrast to the traditional quasi-one-dimensional model. In this case, therefore, one cannot simply integrate the transverse wave vector $k_{\mathrm{t}}$ to obtain the tunneling current, as Zhang et al. did [8]. In short, our $4 \times 4$ transfer matrix calculations are exact within the free electron model and also applicable to both double and multiple barrier junctions as well as arbitrary spin alignment between ferromagnets. Further computational details will be given elsewhere [10].

In this paper, we report the key results of our studies of the $\mathrm{MR}$ of a $\mathrm{Fe} /$ nonmagnetic insulator/Fe junction. The parameters used for $\mathrm{Fe}$ are exchange field $\left(h_{\mathrm{Fe}}\right) 1.9 \mathrm{eV}$, Fermi energy $\left(E_{\mathrm{f}}\right) 2.6 \mathrm{eV}$ and effective mass $\left(m_{\mathrm{Fe}}^{*}\right) 1[11]$. The insulator barrier height $\left(V=V_{\mathrm{I}}-V_{\mathrm{Fe}}\right)$ is $3.6 \mathrm{eV}$ (i.e., $1 \mathrm{eV}$ above $\left.E_{\mathrm{f}}\right)$ [4] and the effective mass $\left(m_{\mathrm{I}}^{*}\right)$ is 0.4 [7]; Fig. 1 shows the tunneling current as a function of bias voltage. As is expected from Julliere model, the calculated tunneling current in parallel spin alignment is larger than anti-parallel one at small bias voltages (below $1 \mathrm{~V})$ (Fig. 1). However, when the bias voltage is larger than $1 \mathrm{~V}$, the calculated current for parallel spin alignment is smaller than antiparallel one. This suggests that the MR would be positive at small voltages but becomes negative at large voltages. In Fig. 2, the MR ratios for three different barrier widths $(d)$ (and the same barrier height of $3.6 \mathrm{eV}$ ) are displayed. For the $10 \AA$ barrier width, the MR ratio is largest $(9 \%)$ at zero bias and decreases as the applied voltage increases, and changes sign at the voltage of about $1 \mathrm{~V}$. The largest negative MR

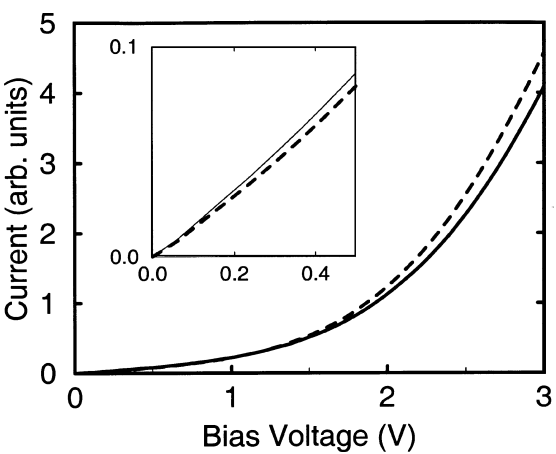

Fig. 1. Tunneling current versus applied voltage for a $10 \AA$ wide barrier. Solid line is for parallel spin alignment and dashed line, for antiparallel alignment. Inset shows the current is larger in parallel alignment at small biases.

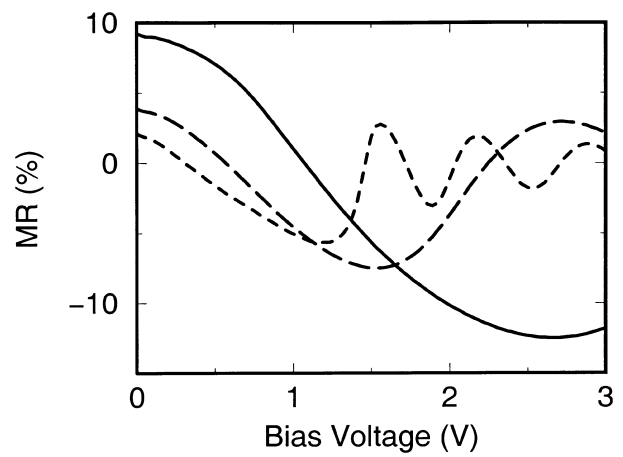

Fig. 2. Magnetoresistance versus applied voltage for different barrier widths. Solid line for a $10 \AA$ barrier; long dashed, $20 \AA$ and dashed, $50 \AA$.

value is $12 \%$. As can be seen from Fig. 2, when longer barriers are used, the MR shows an oscillatory behavior as a function of bias voltage. This is especially pronounced for the barrier width of $50 \AA$ (the dashed curve in Fig. 2). Fig. 2 also shows that the longer the barrier, the short the oscillating period. The negative MR and its oscillatory behavior can be traced back to the nonmonotonical, fluctuating behavior of the transmission coefficient along the longitudinal energy coordinate. When the transmission coefficient is integrated to get the current, this interesting feature survives. This is the central result of this paper. It should be emphasized that both the negative MR and its oscillatory behavior are there in the free electron model and it is not necessary to invoke other elaborate mechanisms to explain them, as did in a previous report [6]. Finally, Fig. 2 shows that in general, the MR of a single barrier junction is positive at zero bias, decreases as the bias voltage increases and eventually becomes negative at certain critical bias, $V_{\mathrm{c}}$. Note that for a single symmetric barrier junction the 
positive MR value at zero bias, though decreases as the barrier width increases, never becomes negative. This finding is in agreement with Ref. [2]. Also, the $V_{\mathrm{c}}$ decreases monotonically with the barrier width but increases with the barrier height. For a typical $\mathrm{Fe} / \mathrm{Al}_{2} \mathrm{O}_{3}$ $(d=10 \AA, V=5.6 \mathrm{eV}) / \mathrm{Fe}$ junction, the calculated $V_{\mathrm{c}}$ is about $2.5 \mathrm{~V}$ which is perhaps too high to be reached experimentally. This could explain why only one recent report [5] on the negative MR so far.

The authors thank Professor C.R. Chang for stimulating discussions. This work is supported by the National Science Council of ROC (NSC 88-2112-M002-0043).

\section{References}

[1] M. Julliere, Phys. Lett. 54 A (1975) 225.

[2] J.C. Slonczewski, Phys. Rev. B 39 (1989) 6995.

[3] J.S. Moodera et al., Phys. Rev. Lett. 74 (1995) 3273.

[4] T. Miyazaki et al., J. Phys. D 31 (1998) 630.

[5] M. Sharma et al., Phys. Rev. Lett. 82 (1999) 616.

[6] S.T. Chui, Phys. Rev. B 55 (1997) 5600.

[7] A.M. Bratkovsky, Phys. Rev. B 56 (1997) 2344.

[8] X. Zhang et al., Phys. Rev. B 56 (1997) 5484.

[9] A. Voskoboynikov et al., Phys. Rev. B 58 (1999) 15397.

[10] S.S. Liu, Master Thesis, National Taiwan University, 1999.

[11] M.B. Stearns, J. Magn. Magn. Mater. 5 (1976) 167. 